\title{
Commentary: Mitochondria: The magic bullet?
}

\author{
Richa Dhawan, MD, MPH, and Mark A. Chaney, MD
}

\author{
From the Department of Anesthesia and Critical Care, University of Chicago Medical Center, Chicago, Ill. \\ Disclosures: Authors have nothing to disclose with regard to commercial support. \\ Received for publication July 15, 2019; accepted for publication July 16, 2019; available ahead of print July 25 , \\ 2019. \\ Address for reprints: Richa Dhawan, MD, MPH, Department of Anesthesia and Critical Care, University of Chi- \\ cago Medical Center, 5841 S Maryland Ave, MC 4028, Chicago, IL 60637 (E-mail: rdhawan@ dacc.uchicago. \\ edu). \\ J Thorac Cardiovasc Surg 2020;160:e31-2 \\ $0022-5223 / \$ 36.00$ \\ Copyright (C) 2019 Published by Elsevier Inc. on behalf of The American Association for Thoracic Surgery \\ https://doi.org/10.1016/j.jtcvs.2019.07.027
}

Since the demonstration of myocardial ischemic preconditioning in dogs by Murry and associates ${ }^{1}$ in 1986, numerous investigators have explored a wide variety of pathways whereby the heart may be protected from ischemic damage. One such notable group at Harvard, led by James D. McCully, extends their pioneering novel therapy, autologous mitochondrial transplantation (AMT), in the article in this issue of the Journal by Guariento and colleagues. ${ }^{2}$ Knowing that ischemia-reperfusion injury initiates a cascade of events ultimately leading to mitochondrial dysfunction-induced myocardial dysfunction, this group previously revealed that myocardial and coronary injection of autologous mitochondria during reperfusion is protective. Here, they demonstrate beneficial effects of preischemic intracoronary AMT. ${ }^{2}$

Their ischemia-reperfusion injury protocol consisted of 30 minutes of ischemia (left anterior descending snare) followed by 120 minutes of reperfusion. A total of 26 pigs were divided into 3 groups: 10 received a single intracoronary AMT before ischemia, 6 received serial intracoronary AMTs before ischemia, and 10 received vehicle intracoronary injections (control). Intensive hemodynamic monitoring occurred, and all animals were eventually killed. Autologous mitochondria were obtained through pectoralis major punch biopsy soon after induction of anesthesia. The results were quite impressive. Relative to controls, both AMT groups exhibited increased coronary blood flow, improved regional myocardial function (segmental/fractional shortening, strain), improved global myocardial function (ejection fraction, diastolic function), and reduced infarct size (histology, transmission electron microscopy). No differences were found between the 2 AMT groups. The results provide strong evidence that preischemic intracoronary AMT attenuates ischemia-reperfusion injury.

Mitochondria have a central role in cellular apoptotic pathways in cardiac myocytes. Numerous proteins and protein receptor-mediated regulatory interactions are involved in controlling mitochondrial dynamics. Reperfusion injury, characterized by an increase in calcium and reactive oxygen species, activates a fragmentation process in mitochondria,

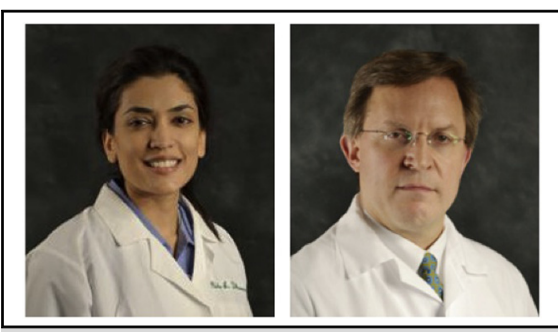

Richa Dhawan, MD, MPH (left), and Mark A. Chaney, MD (right)

\section{Central Message \\ Autologous mitochondrial transplantation ameliorates ischemia-reperfusion injury after myocardial insult in animal models and holds potential for clinical trials.}

See Article page e15. resulting in loss of normal regulation. ${ }^{3}$ Ideally, an intervention would inhibit a specific mechanistic route to prevent this type of dysregulation; however, the sheer complexity of interactions makes this difficult. Guariento and colleagues, ${ }^{2}$ in a series of clever studies, have bypassed this problem by introducing complete healthy mitochondria into damaged tissue. Although it is unclear how they exert their beneficial effect, transplanted mitochondria ameliorate myocardial injury.

AMT has tremendous potential for translation into human patients; however, studies on AMT are limited to animal models, with a single clinical trial. McCully and colleagues $^{4}$ described AMT a decade ago, and that group ${ }^{5}$ went on to conduct the first successful in vivo trial in pediatric patients with cardiac disease. This same group developed techniques for rapid mitochondrial isolation, making real-time application feasible, as in their current study. ${ }^{2}$

Mitochondria inherently represent the perfect cellular organelle for autotransplantation, because of their unique attributes. For instance, mitochondria continue to function independently from the host cell, they are not arrhythmogenic (unlike myoblasts), and autotransplantation mitigates the possibility of an autoimmune reaction. ${ }^{6}$ Future clinical trials would have to demonstrate rapid acquisition of functional mitochondria in real time, clarify the effective dose and delivery method, clarify the optimal timing of treatment, and perform a risk-benefit analysis. The applicability of AMT is widespread in ischemic cardiac disease, and it could become a powerful adjunct in clinical treatment of the disease. 


\section{References}

1. Murry CE, Jennings RB, Reimer KA. Preconditioning with ischemia: a delay of lethal cell injury in ischemic myocardium. Circulation. 1986;74:1124-36.

2. Guariento A, Blitzer D, Doulamis I, Shin B, Moskowitzova K, Orfany A, et al. Preischemic autologous mitochondrial transplantation by intracoronary injection for myocardial protection. J Thorac Cardiovasc Surg. 2020;160:e15-29.

3. Nan J, Zhu W, Rahman MS, Liu M, Li D, Su S, et al. Molecular regulation of mitochondrial dynamics in cardiac disease. Biochim Biophys Acta. 2017;1864: 1260-73.
4. McCully JD, Cowan DB, Pacak CA, Toumpoulis IK, Dayalan H, Levitsky S. Injection of isolated mitochondria during early reperfusion for cardioprotection. Am J Physiol Heart Circ Physiol. 2009;296:H94-105.

5. Emani SM, Piekarski D, Harrild PJ, Del Nido PJ, McCully JD. Autologous mitochondrial transplantation for dysfunction after ischemia-reperfusion injury. $J$ Thorac Cardiovasc Surg. 2017;154:286-9.

6. Masuzawa A, Black KM, Pacak CA, Ericsson M, Barnett RJ, Drumm C, et al. Transplantation of autologously derived mitochondria protects the heart from ischemia-reperfusion injury. Am J Physiol Heart Circ Physiol. 2013;304:H966-82. 